\title{
Anti-aging effects of melatonin on the myocardial mitochondria of rats and associated mechanisms
}

\author{
XIN-HONG GUO $^{1 *}$, YAN-HUA LI ${ }^{*}$, YU-SHENG ZHAO ${ }^{1}$, YONG-ZHI ZHAI ${ }^{2}$ and LI-CHENG ZHANG ${ }^{3}$ \\ ${ }^{1}$ Department of Cardiology, Institute of Geriatric Cardiology; ${ }^{2}$ Emergency Department; ${ }^{3}$ Department of Orthopedics, \\ Institute of Orthopedics, Chinese People's Liberation Army General Hospital, Beijing 100853, P.R. China
}

Received October 27, 2015; Accepted September 15, 2016

DOI: $10.3892 / \mathrm{mmr} .2016 .6002$

\begin{abstract}
The present study investigated the anti-aging effects of melatonin on the myocardial mitochondria of D-galactose-aged rats and associated mechanisms. A total of 30 male Sprague-Dawley (SD) rats were randomly divided into three equal groups: An accelerated aging group that received $125 \mathrm{mg} / \mathrm{kg} /$ day D-galactose; a melatonin-treated group of D-galactose-aged rats that received $10 \mathrm{mg} / \mathrm{kg} /$ day melatonin; and a control group receiving normal saline. ATP, ADP and AMP levels in the left ventricular myocardium of rats were determined by high performance liquid chromatography and the total adenylic acid number (TAN) was subsequently calculated. Bax, Bcl-2, and cytochrome c (cyt-c) protein expression levels in myocardial mitochondria and cytoplasm were quantified by western blot analysis. In the melatonin-treated group, ATP levels were significantly higher when compared with the untreated control group and the accelerated-ageing group (0.068 vs. 0.052 and $0.058 ; \mathrm{P}=0.002$ and $\mathrm{P}=0.045$, respectively), and TAN was significantly increased in the melatonin-treated group when compared with controls $(\mathrm{P}=0.011)$. In addition, cyt-c levels in the cytoplasm, but not in the mitochondria, were significantly higher in the accelerated-aging group compared with the control and melatonin-treated groups $(\mathrm{P}=0.001$ and $\mathrm{P}=0.002$, respectively). Bcl-2 and Bax ratios were significantly higher in the control and melatonin-treated groups when compared with the accelerated-aging group ( $\mathrm{P}=0.004$ and $\mathrm{P}=0.032$, respectively).
\end{abstract}

Correspondence to: $\mathrm{Dr}$ Yu-Sheng Zhao, Department of Cardiology, Institute of Geriatric Cardiology, Chinese People's Liberation Army General Hospital, 28 Fuxing Road, Beijing 100853, P.R. China

E-mail: yushengzh5@sina.com

*Contributed equally

Abbreviations: AGEs, advanced glycation end products; cyt-c, cytochrome c; EC, energy charge; SD, Sprague-Dawley; TAN, total adenylic acid number

Key words: aging, Bax, Bcl-2, cytochrome c, mitochondria, melatonin, myocardium
These results suggest that melatonin exhibits a protective effect on mitochondrial function in a rat model of accelerated aging.

\section{Introduction}

Aging is a complex physiological process involving the progressive, irreversible accumulation of oxidative damage sustained by cellular components during aerobic respiration $(1,2)$. Accelerated aging and death in senescence-accelerated mice (SAM) is attributed to hyperoxidation (3). Mitochondria, the primary source of reactive oxygen species (ROS) and oxidative damage, produce ROS during cellular respiration, which leads to impaired physiological function, increased incidence of disease and reduced lifespan (4). During respiration, mitochondria produce the primary cellular energy component ATP, which regulates cellular metabolism and modulates cell apoptosis (5). Aging bodies are characterized by abnormal energy metabolism, including decreased mitochondrial ATP generation, increased ROS generation and subsequent damage or disease (6). Neurodegenerative diseases may arise from increased mitochondrial dysfunction, oxidative stress and apoptosis (7). Cardiac function is particularly susceptible to ROS production, mitochondrial DNA damage and respiratory chain impairment (8). Mitochondrial control of cellular respiration is associated with cardiac function insufficiency and remodeling of the prostrated heart (9). ATP is essential for myocardial contraction and electrophysiology and maintaining normal cardiac function during myocardial aging (10), while declining cardiac function is marked by severe mitochondrial dysfunction and ATP deficiency $(6,11,12)$.

Antioxidants reduce oxidative damage arising from abnormal mitochondrial metabolism (13). The oxidative stress theory of aging describes antioxidants as ROS scavengers that enable the prevention and repair of oxidative damage, which may reduce age-associated illness (4). Melatonin, which is synthesized from tryptophan primarily in the pineal gland, is a free radical scavenger, and is the primary anti-oxidative defense against reactive hydroxyl radicals $(14,15)$. As a pleiotropic molecule, melatonin exerts multiple beneficial effects on age-related physiological functions, including metabolic sensing, modulation and proliferation of mitochondria, the antioxidative protection of biomolecules and anti-inflammatory actions (16). In vitro and in vivo studies have demonstrated 
that melatonin effectively counteracts oxidative stress and oxidative damage (17-19). Melatonin demonstrates obvious anti-aging effects, including protecting the mitochondria of young and aged rats $(20,21)$, resisting mitochondrial dysfunction in SAM mice (22), and normalizing the energy status of heart mitochondria and increasing ATP levels in SAM mice with mitochondrial oxidative stress (2). Loss of melatonin production has long been associated with aging (23). Young hamsters exhibited an 8-fold increase in pineal melatonin levels in the dark phase of a 24-h period, while aging hamsters demonstrated no increase in nocturnal melatonin production, indicating a marked reduction in pineal biosynthetic activity associated with aging (24). Similarly, melatonin levels in young, middle-aged and aged female rats sacrificed during light and dark periods revealed low melatonin levels in the pineal glands of all animals sacrificed during light periods (25). By contrast, marked increases (12-fold) in the melatonin levels of young animals compared with older rats (6-fold) were observed during dark periods, which indicates a notable reduction in pineal melatonin production with age (25). Loss of melatonin is suggested to be an indicator of ageing (23). However, although melatonin may improve temporal organization in advanced age and demonstrates beneficial effects on sleep and age-related diseases, its protective effects against age-related decline are not fully understood (26).

In the present study, the authors hypothesized that melatonin may function in resistance to myocardial aging by protecting the mitochondria, which could be demonstrated by measuring cellular energy stores in aged rats. An accelerated aging model was established by treating rats with D-galactose as described previously (27). In China, D-galactose-induced aging is a common method used to generate animal models of aging and in studies of age-associated pharmacotherapy (27). D-galactose forms advanced glycation end products (AGEs) in vivo, and a comparison of D-galactose-aged and AGEs-aged mice demonstrated that both models resembled aged mice, suggesting that advanced glycation may be part of the D-galactose aging mechanism (28). The aim of the present study was to investigate the anti-aging effects of melatonin on the myocardial mitochondria of D-galactose aged rats, and to explore associated mechanisms.

\section{Materials and methods}

Animals. A total of 30 healthy male Sprague-Dawley (SD) rats (age, 3 months; weight, 300-350 g) were purchased from the Laboratory Animal Center of the Academy of Military Medical Sciences (Beijing, China). All investigations were performed in accordance with the Guide for the Care and Use of Laboratory Animals of the Institutional Review Board of Chinese People's Liberation Army (PLA) General Hospital (identification no. 2008-X1-70). Rats had ad libitum access to a commercial rat chow diet and tap water. The animals were housed four per cage, and maintained at $22 \pm 2^{\circ} \mathrm{C}$ and $50-60 \%$ relative humidity, under a $12-\mathrm{h}$ light/dark cycle. The Institutional Review Board of Chinese PLA General Hospital reviewed and approved the study.

Grouping. All SD rats were randomly divided into 3 groups of 10 rats as follows: i) An accelerated aging group (D group) in which 3-month-old rats were subject to accelerated ageing with daily subcutaneous injections of $125 \mathrm{mg} / \mathrm{kg}$ D-galactose (Beijing MENGYIMEI Biology Co., Ltd., Beijing China) for 6 weeks; ii) a melatonin-treated group ( $\mathrm{M}$ group) in which 3-month old rats were aged with D-galactose administration $(125 \mathrm{mg} / \mathrm{kg} /$ day) and treated with $10 \mathrm{mg} / \mathrm{kg} /$ day melatonin (Shanghai Hengyuan Biological Technology Co., Ltd., Shanghai, China) for 6 weeks; and iii) a control group (C group) in which 3-month-old rats were treated with equal volume of normal saline via daily subcutaneous and intraperitoneal injections for 6 weeks.

Establishing the accelerated aging rat model. A total of 10 three-month-old rats were subject to accelerated ageing by administration of D-galactose $(125 \mathrm{mg} / \mathrm{kg} /$ day diluted in normal saline) delivered daily at 3:00 p.m. by subcutaneous injection for 6 weeks, as described previously $(27,28)$. This method is based on the metabolic disturbance theory of aging, which involves inducing galactose overload via a high galactose diet or injection of galactose, in order to promote subacute aging similar to natural aging effects whereby D-galactose forms AGEs in vivo. Successful and sufficient aging of rats in the present study was characterized by the development of torpescence, sluggishness, grey hair or trichomadesis of rats in the accelerated aging group ( $\mathrm{D}$ group) compared to the untreated control rats.

Materials and sample preparation. Rats in all three groups were sacrificed at the end of the 6-week treatment period, when they were 4.5 months old. Briefly, rats were anaesthetized with urethane via intraperitoneal injection, and the hearts were removed and subsequently flushed with saline as described previously (29). The free walls of the left ventricles were then excised immediately. For each sample, a portion of myocardial tissue was first placed into $1 \mathrm{~mol} / 1 \mathrm{HClO}_{4}$, then transferred into freezing tubes and preserved in liquid nitrogen until required for evaluation. The left ventricular myocardium was weighed precisely, and $100 \mathrm{mg}$ myocardial tissue was divided into pieces using eye scissors and placed into a pre-cooled tissue grinder, to which $1 \mathrm{~mol} / 1 \mathrm{HClO}_{4}$ at $4^{\circ} \mathrm{C}$ was added at the ratio of $1 \mathrm{ml} / 100 \mathrm{mg}$. To obtain homogenate, pieces of pre-cut tissue were homogenized in an ice bath for 2 min using a CAT Scientific X120 Handheld Homogenizer Drive $(3000 \mathrm{r} / \mathrm{min}$ motor-driven homogenizer; PolyScience, Niles, IL, USA). The homogenized tissues were centrifuged for $10 \mathrm{~min}$ at $4^{\circ} \mathrm{C}$ and $6099 \times \mathrm{g}$. For each sample, $800 \mu \mathrm{l}$ supernatant was transferred into a fresh $1.5 \mathrm{ml}$ Eppendorf tube, to which $2 \mathrm{~mol} / 1 \mathrm{KH}_{2} \mathrm{PO}_{4}$ and $5 \mathrm{~mol} / \mathrm{KOH}$ were added. Samples where then vigorously shaken on a vibrator, the $\mathrm{pH}$ was adjusted to 7.0 , cooled to $4^{\circ} \mathrm{C}$ and then centrifuged for $10 \mathrm{~min}$ at $4^{\circ} \mathrm{C}$ and $6099 \times \mathrm{g}$. For each sample, $200 \mu \mathrm{l}$ of the supernatant was transferred into a $0.5 \mathrm{ml}$ Eppendorf tube for testing by high performance liquid chromatography (HPLC). The remaining section of myocardial tissue was subjected to freezing with liquid nitrogen to extract myocardial mitochondria proteins, in order to observe differences in the amounts of total mitochondrial proteins among groups, and to measure alterations in the protein expression levels of Bax, Bcl-2 in the mitochondria and cytochrome c (cyt-c) in the cytoplasm. 
HPLC evaluation. An Agilent 1100 Series HPLC system (Agilent Technologies, Inc., Santa Clara, CA, USA) was used. The chromatographic analysis for the determination of ATP, ADP and AMP was performed using a Zorbax, C (18) column (150x4.6 mm; $5 \mu \mathrm{m}$; Agilent Technologies, Inc.). The mobile phase consisted of phosphate buffer (solvent $\mathrm{A}$ : $6 \mathrm{mmol} / 1 \mathrm{Na}_{2} \mathrm{HPO}_{4}$ and $44 \mathrm{mmol} / 1 \mathrm{NaH}_{2} \mathrm{PO}_{4}, \mathrm{pH} \mathrm{5.8)}$ and methanol (solvent $\mathrm{B}$ ), $76 / 24$, comprising the ion pair reagent A $5 \mathrm{mmol} / \mathrm{l}$. For each sample, $20 \mu \mathrm{l}$ was added to the sample injector of the HPLC instrument to measure the levels of ATP, ADP and AMP at a detection wavelength of $259 \mathrm{~nm}$. The solvent gradient elution conditions were $100 \%$ A, $0 \%$ B for $0 \mathrm{~min} ; 95 \% \mathrm{~A}, 5 \% \mathrm{~B}$ for $2 \mathrm{~min} ; 80 \% \mathrm{~A}, 20 \% \mathrm{~B}$ for $4 \mathrm{~min}$; $75 \% \mathrm{~A}, 25 \% \mathrm{~B}$ for $5 \mathrm{~min}$; and $100 \% \mathrm{~A}, 0 \% \mathrm{~B}$ for $6 \mathrm{~min}$ at a flow-rate of $1.0 \mathrm{ml} / \mathrm{min}$, and the column was maintained at a temperature of $25^{\circ} \mathrm{C}$ throughout the study. Levels of ATP, ADP and AMP in rat myocardial tissue were detected using the external standard method (30), and the total adenylic acid number (TAN) was calculated using the following formula: $\mathrm{TAN}=\mathrm{ATP}+\mathrm{ADP}+\mathrm{AMP}$. The energy state of myocytes was indicated by the energy charge (EC), using the computational formula $\mathrm{EC}=(\mathrm{ATP}+1 / 2 \mathrm{ADP}) / \mathrm{TAN}$, as described previously (31).

Mitochondria extraction. Briefly, $100 \mathrm{mg}$ of myocardial tissue was weighed and the mitochondria were extracted using a mitochondria/cytosol fractionation kit (cat no. ab65320; Abcam, Cambridge, MA, USA). Tissues were divided into $0.5 \mathrm{~cm}^{3}$ fragments with scissors and placed into a small volume glass homogenizer as described previously (32). The total volume of the tissue fragments was estimated, and $1.5 \mathrm{ml}$ pre-cooled mitochondria/cytosol fractionation buffer (Mito-Cyto Buffer; Mitochondria Isolation kit; Applygen Technologies Inc., Beijing, China) was added. The tissues were placed into a grinder and ground 20 times. The tissue homogenate was then transferred to a centrifuge tube and centrifuged at $27 \times g$ for $5 \mathrm{~min}$ at $4^{\circ} \mathrm{C}$. Cell nuclei, membrane fragments and non-disintegrated cells were pelleted. The supernatant liquid was collected, transferred to a new centrifuge tube and centrifuged at $27 \times \mathrm{g}$ for $5 \mathrm{~min}$ at $4^{\circ} \mathrm{C}$. The precipitate was discarded, and the supernatant liquid was transferred to a new centrifuge tube, and centrifuged at $6099 \times \mathrm{g}$ for $10 \mathrm{~min}$ at $4^{\circ} \mathrm{C}$. The supernatant fluid containing cytoplasmic components was then transferred to a new centrifuge tube, leaving behind the mitochondrial pellet. To wash the mitochondrial pellet, liquid and fragments adhering to the inner wall of the tube were first removed and $0.2 \mathrm{ml}$ Mito-Cyto Buffer was added to re-suspend the mitochondria precipitate, which was centrifuged at $6099 \times \mathrm{g}$ for $10 \mathrm{~min}$ at $4^{\circ} \mathrm{C}$. The supernatant was discarded and the mitochondria precipitate was re-suspended with Mito-Cyto Buffer (50 $\mu \mathrm{l} / 100 \mathrm{mg}$ tissue). After detecting protein concentration using the Bradford method (33), unused mitochondria precipitates were preserved immediately at $-70^{\circ} \mathrm{C}$. The concentration of samples was determined using a Bradford Protein assay kit (Bio-Rad Laboratories, Inc., Hercules, CA, USA) according to the manufacturer's instructions.

Bax, Bcl-2, and cyt-c protein levels in mitochondria and cytoplasm. Samples $(50 \mu \mathrm{g})$ were loaded onto $10 \%$
SDS-PAGE gels with $8 \mu 1$ prestained and $4 \mu 1$ illuminable protein molecular weight markers. Electrophoresis was performed and proteins were transferred to polyvinyldene difluoride (PVDF) membranes. Membranes were blocked with $5 \%$ skimmed milk for $1 \mathrm{~h}$ at room temperature, and incubated overnight at $4^{\circ} \mathrm{C}$ with the following primary antibodies diluted in 5\% skimmed milk: Rabbit anti-Bax $(1: 1,500$; catalog no. sc-6236), mouse anti-Bcl-2 (1:1,000; catalog no. sc-7382), goat anti-cyt-c $(1: 2,000$; catalog no. sc-8385) and rabbit anti-GAPDH (1:2,000; catalog no. sc-25778), obtained from Santa Cruz Biotechnology, Inc. (Dallas, TX, USA). Membranes were washed and incubated with horseradish peroxidase-conjugated goat anti-rabbit $\operatorname{IgG}(1: 2,000$; catalog no. sc-2004), goat anti-mouse $\operatorname{IgG}(1: 2,000$; catalog no. sc-2005) or donkey anti-goat IgG (1:2,000; catalog no. sc-2033) secondary antibodies, obtained from Santa Cruz Biotechnology, Inc., for $1 \mathrm{~h}$ at $37^{\circ} \mathrm{C}$. Proteins were detected using an Enhanced Chemiluminescence reagent (Bio-Rad Laboratories, Inc.) by adding Detection Reagent 2 (1:1 concentration) to each $6 \times 9 \mathrm{~cm}$ PVDF membrane, allowing liquid to cover the film surface, and incubating at room temperature for $1 \mathrm{~min}$. The reaction liquid was drained and PVDF film was isolated with preservative film and placed in an exposure box for compressed exposure in the darkroom before photos were taken. Gel images were analyzed using Quantity One software version 4.62 (Bio-Rad Laboratories Inc.), and the results were presented as integral absorbance values.

Statistical analysis. Data are presented as mean \pm standard deviation. Differences among the three treatment groups were tested using a one-way analysis of variance (ANOVA). The least significant difference post-hoc comparisons test was performed when the corresponding one-way ANOVA test reached statistical significance. Differences were considered to be statistically significant when $\mathrm{P}<0.05$. All statistical analyses were performed using SPSS (version, 15.0; SPSS Inc., Chicago, IL, USA).

\section{Results}

Comparison of adenylic acid content and EC in rat myocardial tissues. No significant differences in AMP and ADP levels among groups were observed (Table IA). The ATP level in the melatonin-treated group (group M) was significantly higher than those of the control (group C) and accelerated-aging (group D) group (0.068 vs. 0.052 and $0.058 \mu \mathrm{mol} / \mathrm{g}$; $\mathrm{P}=0.002$ and $\mathrm{P}=0.045$, respectively). The TAN level of the melatonin-treated group $(\mathrm{M})$ was also higher when compared with the control and accelerated-aging group ( 0.160 vs. 0.133 and $0.142 \mu \mathrm{mol} / \mathrm{g}$, respectively; Table IA), but only the difference between the melatonin-treated group and control group reached statistical significance $(\mathrm{P}=0.011)$. The energy charge (EC) of the melatonin-treated group was increased, however this did not reach statistical significance when compared with the control and accelerated-ageing groups ( 0.570 vs. 0.529 and $0.561 \mu \mathrm{mol} / \mathrm{g}$; Table IA and Fig. 1).

Comparison of Bax, Bcl-2 and cyt-c protein levels in myocardial tissue mitochondria and cytoplasm. Cyt-c protein 
Table I. Comparison of adenylic acid levels and cyt-c, Bax and Bcl-2 protein expression among the treatment groups.

A, Adenylic acid content of myocardial tissues

\begin{tabular}{lccc}
\hline Adenylic acid/EC & Group C $(\mu \mathrm{mol} / \mathrm{g})$ & Group M $(\mu \mathrm{mol} / \mathrm{g})$ & Group D $(\mu \mathrm{mol} / \mathrm{g})$ \\
\hline AMP & $0.044 \pm 0.005$ & $0.044 \pm 0.011$ & $0.041 \pm 0.009$ \\
ADP & $0.032 \pm 0.007$ & $0.037 \pm 0.006$ & $0.035 \pm 0.003$ \\
ATP & $0.052 \pm 0.009$ & $0.068 \pm 0.012^{\mathrm{a}}$ & $0.058 \pm 0.008^{\mathrm{b}}$ \\
TAN & $0.133 \pm 0.027$ & $0.160 \pm 0.020^{\mathrm{a}}$ & $0.142 \pm 0.017$ \\
EC & $0.529 \pm 0.056$ & $0.570 \pm 0.049$ & $0.561 \pm 0.028$ \\
\hline
\end{tabular}

B, Cyt-c protein expression in myocardial mitochondria and cytoplasm

\begin{tabular}{lrrr}
\hline Fraction & Group C (IOD) & Group M (IOD) & Group D (IOD) \\
\hline Cytoplasmic & $2.192 \pm 0.164$ & $2.228 \pm 0.392$ & $2.892 \pm 0.609^{\mathrm{a}, \mathrm{b}}$ \\
Mitochondria & $1.067 \pm 0.209$ & $0.999 \pm 0.226$ & $1.055 \pm 0.288$ \\
Cytoplasmic/mitochondria & $2.134 \pm 0.488$ & $2.272 \pm 0.359$ & $2.838 \pm 0.635^{\mathrm{a}, \mathrm{b}}$ \\
\hline
\end{tabular}

C, Protein expression in myocardial mitochondria

\begin{tabular}{lccc}
\hline Protein & Group C (IOD) & Group M (IOD) & Group D (IOD) \\
\hline Bax & $0.809 \pm 0.178$ & $0.674 \pm 0.099$ & $0.687 \pm 0.158$ \\
Bcl-2 & $1.217 \pm 0.218$ & $0.959 \pm 0.166^{\mathrm{a}}$ & $0.780 \pm 0.194^{\mathrm{a}, \mathrm{b}}$ \\
Bcl-2/Bax & $1.540 \pm 0.293$ & $1.435 \pm 0.251$ & $1.162 \pm 0.265^{\mathrm{a}, \mathrm{b}}$ \\
\hline
\end{tabular}

Data are expressed as the mean \pm standard deviation. ${ }^{\text {a }}<0.05$ vs. group $\mathrm{C}$; ${ }^{\mathrm{b}} \mathrm{P}<0.05$ vs. group $\mathrm{M}$. Cyt-c, cytochrome c; EC, energy charge; TAN, total adenylic acid number; Group C, untreated control group; Group M, melatonin plus D-galactose-treated rat group; Group D, D-galactose-treated rat group; IOD, integral optical density.

expression in the cytoplasm was significantly higher in the accelerated-aging group compared with the control and melatonin-treated groups (2.892 vs. 2.192 and 2.228; $\mathrm{P}=0.001$ and $\mathrm{P}=0.002$, respectively; Table $\mathrm{IB})$. The representative western blot images are shown in Fig. 2A. No significant differences in the protein expression levels of cyt-c in mitochondria were observed among groups (Table IB). The representative western blot images are shown in Fig. 2B. The cytoplasmic/mitochondrial ratio of cyt-c expression was significantly higher in the accelerated aging group compared with that of the control and melatonin-treated groups (2.838 vs. 2.134 and 2.272; $\mathrm{P}=0.004$ and $\mathrm{P}=0.019$, respectively; Table IB).

Rats in the melatonin-treated and accelerated-aging groups demonstrated a significant reduction in Bcl-2 expression levels when compared with the control group ( 0.959 and 0.780 vs. 1.217; $\mathrm{P}=0.006$ and $\mathrm{P}<0.001$, respectively; Table IC). The representative western blot images are shown in Fig. 3A. Bcl-2 levels in the accelerated-aging group were significantly lower than in the melatonin-treated group ( 0.780 vs. $0.959 ; \mathrm{P}=0.0497$; Table IC). No significant differences in Bax protein levels were observed among groups. The representative western blot images are shown in Fig. 3B. The ratio between Bcl-2 and Bax in the control group and melatonin-treated group were significantly higher than that in the accelerated-aging group (1.540 and 1.435 vs. $1.162 ; \mathrm{P}=0.004$ and $\mathrm{P}=0.032$, respectively; Table IC).

\section{Discussion}

The results of the present study suggest that melatonin exerts a beneficial anti-aging effect on myocardial tissues in rats via several possible mechanisms. ATP levels were significantly higher in aged rats receiving melatonin when compared with untreated aged rats and normal controls. TAN levels were higher in the melatonin-treated group when compared with the other groups, and were significantly higher than the controls. The protein expression levels of cyt-c in the cytoplasm was significantly higher in the accelerated aging group when compared with the other two groups, however its expression in the mitochondria was not significantly increased. Whilst Bcl-2 protein levels were significantly lower in the melatonin-treated and accelerated-aging groups compared with the controls, Bax protein expression was not significantly different among the groups. However, Bcl-2 and Bax ratios were significantly higher in the control and melatonin-treated groups compared with the accelerated-aging group.

The results of the present study are consistent with those of previous studies that have demonstrated efficacy of short- and long-term melatonin treatment in preventing age-associated mitochondrial oxidative stress (6). Age-dependent oxidative alterations in rat heart mitochondria were associated with reduced activity of the electron transport chain complex 


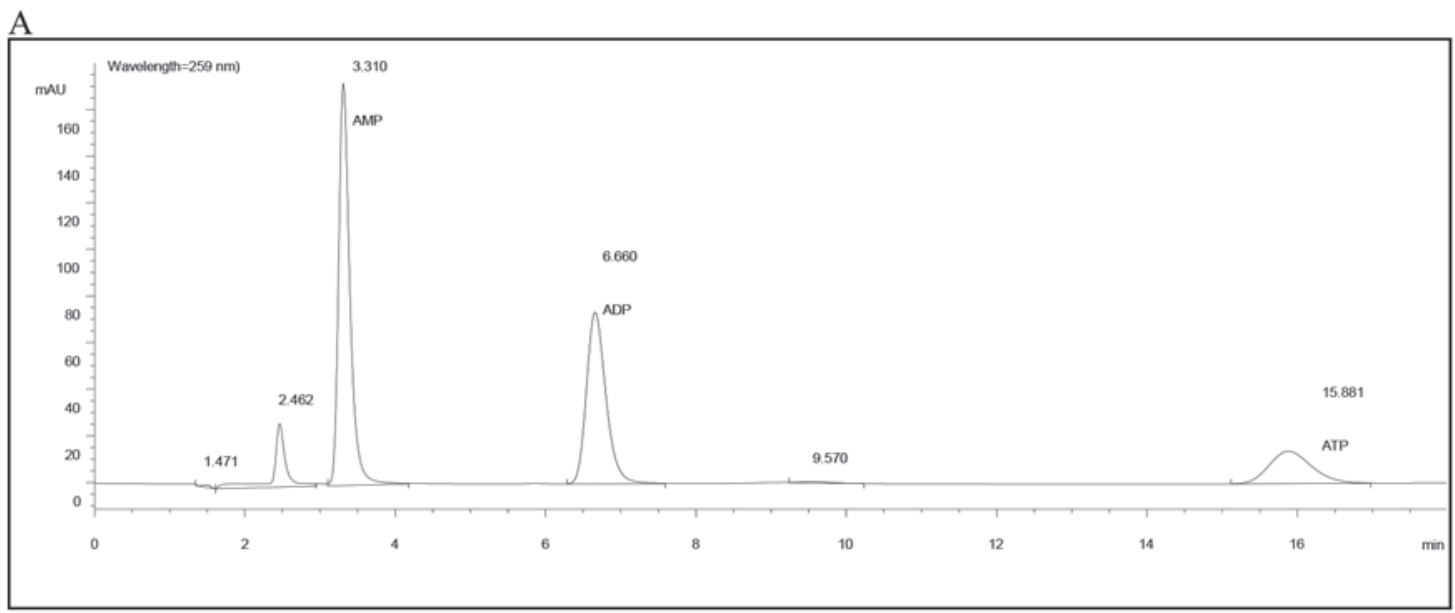

B
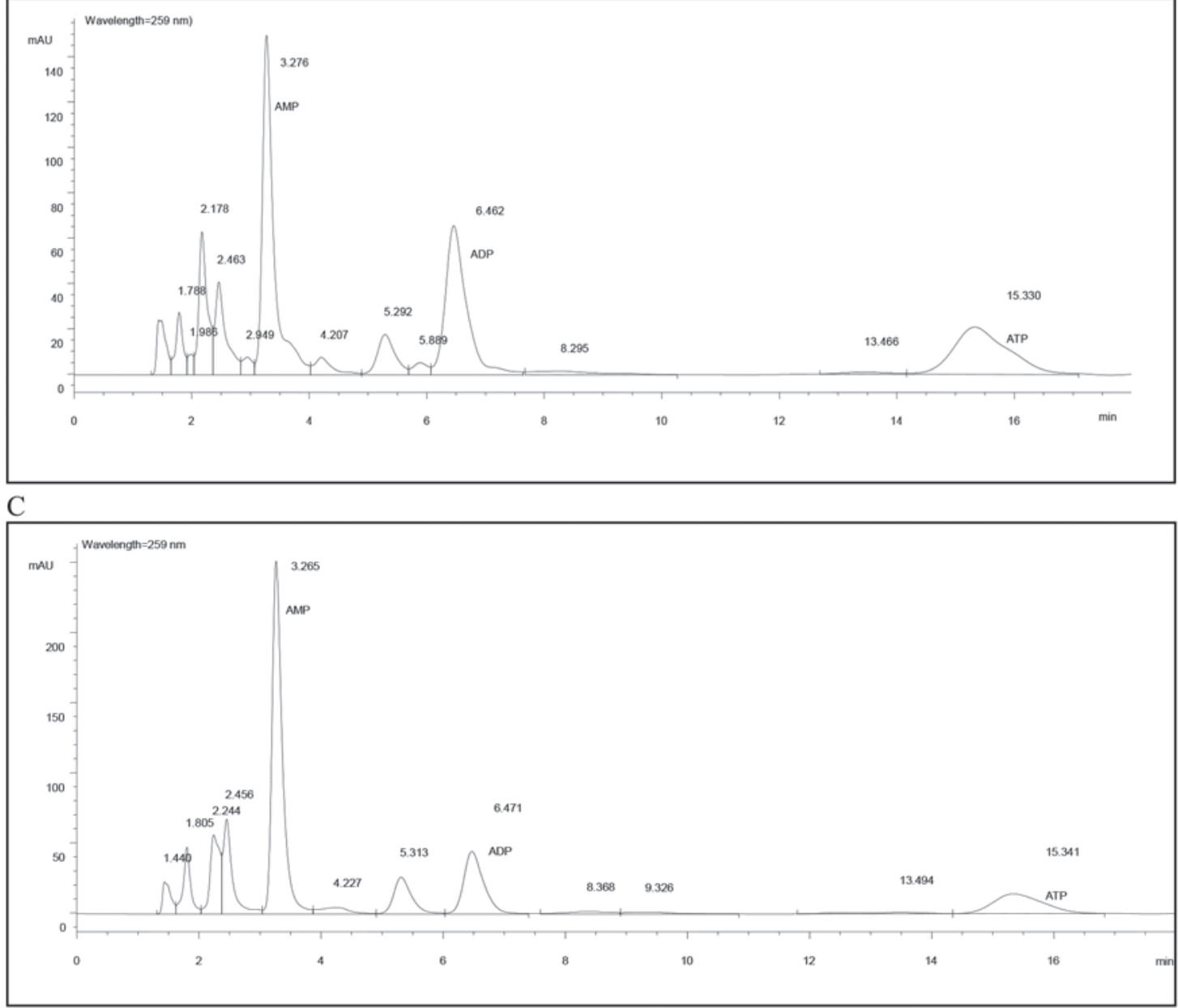

Figure 1. High performance liquid chromatography graphs of AMP, ADP and ATP standards in the myocardium. (A) Control group (group C). (B) melatonin-treated group (group M). (C) accelerated aging group (group D). mAU, milli absorbance units.

and reduced ATP levels; while ATP levels increased and the bioenergetic status was restored following chronic melatonin treatment (2). It has been demonstrated that JAK2/STAT3 activation by melatonin attenuates mitochondrial oxidative damage induced by myocardial ischemia/reperfusion (34). Similarly, melatonin treatment counteracted increased lipid peroxidation and reduced glutathione in the mitochondria of SAM mice (6). In addition, the function of brain mitochondria was maintained by melatonin therapy by preventing impairment of the mitochondria and increasing ATP production (35). Melatonin treatment increased ATP production in rat brain and liver mitochondria in controls and counteracted cyanide-induced inhibition of ATP synthesis, suggesting that mitochondrial homeostasis may be integral to the anti-aging and neuroprotective properties of melatonin (36). 
$\mathbf{A}$

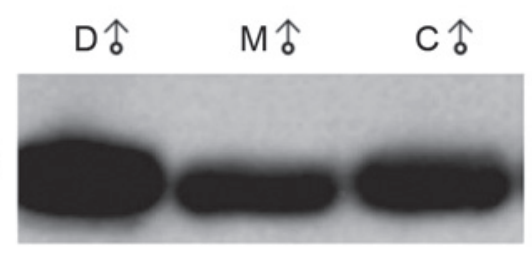

GAPDH

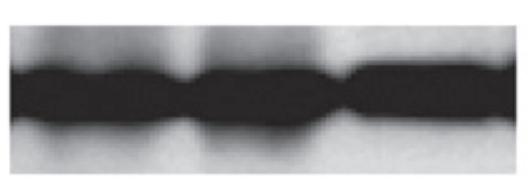

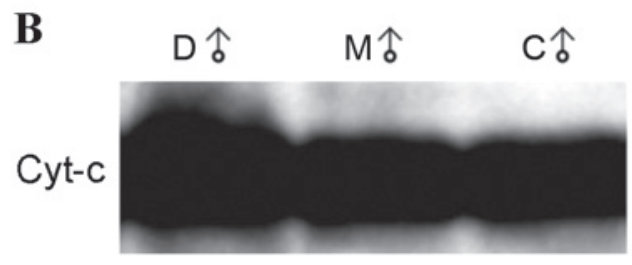

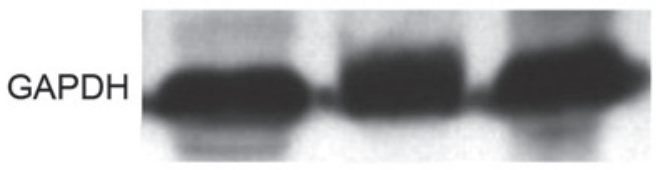

Figure 2. Representative western blot images of cyt-c in the cytoplasm and mitochondria of melatonin-treated rats subjected to D-galactose-induced accelerated ageing. (A) Cyt-c protein expression in cytoplasm was higher in the D group compared with the M group or the C group. (B) No differences in the mitochondrial expression of cyt-c among all groups were observed. Cyt-c, cytochrome c; D, D-galactose-treated rats; M, melatonin plus D-galactose-treated rats; $\mathrm{C}$, untreated controls.
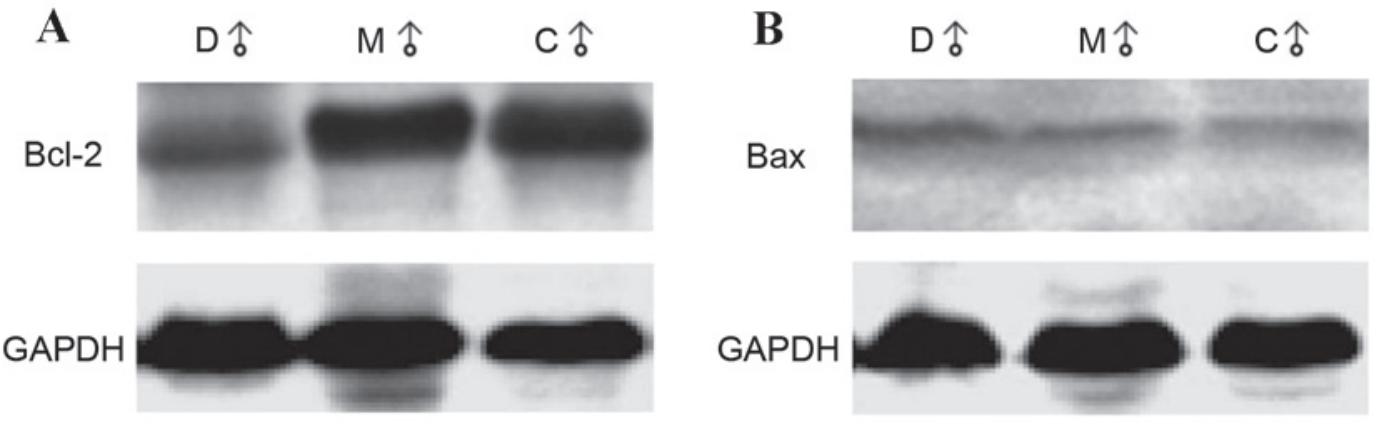

Figure 3. Representative western blot images of Bcl-2 and Bax expression in the mitochondria of melatonin-treated rats subjected to D-galactose-induced accelerated ageing. (A) Rats in M and D-groups exhibited lower Bcl-2 levels compared with the control group. (B) No differences in Bax protein levels were observed among groups. D, D-galactose-treated rats; M, melatonin plus D-galactose-treated rats; C, untreated controls.

In the present study, the energy status of myocytes (by EC measurement) was increased in the melatonin-treated group; however, this did not reach statistical significance. ATP, ADP and AMP constitute a cellular energy-containing state in which an abundance of cellular ATP indicates higher energy levels, while an abundance of cellular AMP suggests lower energy levels (37). Mitochondrial production of ATP is a multi-enzyme process requiring oxygen; however, when ROS inhibits the electron transport chain, mitochondria have insufficient oxygen and ATP decreases (5). This may provide an explanation for the aging mechanism and the development of age-associated degenerative disease. In the present study, ATP and TAN values were significantly higher in melatonin-treated rats compared with controls and those subjected to accelerated ageing. EC values were also higher in melatonin-treated rats when compared with the other groups, however this did not reach statistical significance. This may be explained by a decrease in mitochondrial function during ageing, possibly accompanied by reduced numbers of mitochondria, precluding the maintenance of energy levels. In a previous study, melatonin was observed to enhance myocardial mitochondria generation in aged myocardium, suggesting that its anti-aging effects may protect the integrity of the mitochondrial structure and maintain the number and function of the mitochondria (38). The antioxidant activity of melatonin may be of particular interest in late-onset neurodegenerative diseases, such as Alzheimer's disease and Parkinson's disease, due to the vulnerability of the nervous system to oxidative damage and neoplastic disease (26). In addition, a previous study suggested that melatonin exerts oncostatic actions against tumor development and growth (39). The results of the present study suggest that the effects of melatonin are most likely to be a consequence of free-radical scavenging activity at the level of the mitochondria. Melatonin metabolites are also free radical scavengers. Galano et al (40) reported continuous protection exerted by melatonin metabolites through a free radical scavenging cascade. Melatonin efficiently protects against oxidative stress via immune-enhancing, anti-inflammatory and homeostatic activities in the mitochondria, as well as inhibiting cancer progression (41).

Cyt-c transfers mitochondrial electrons from the cyt-c reductase complex to the cyt-c oxidase complex, suggesting that intracellular cyt-c reduction leads to electron transfer dysfunction, which subsequently leads to ROS generation and reduced ATP production (42). Release of cyt-c from the mitochondria to cytoplasm initiates the apoptosis process (43). In the present study, no significant differences in the protein expression levels of cyt-c in the mitochondria and cytoplasm among groups were observed; however the ratio of cyt-c in cytoplasm vs. the mitochondria in untreated aged rats was significantly higher than the ratio in control and melatonin-treated groups. This suggests that melatonin may maintain the electron transport chain by elevating cyt-c thereby preserving normal oxidative phosphorylation in the mitochondria and decreasing electron leakage. This 
mechanism, along with suppressed transfer of cyt-c from the mitochondria to the cytoplasm, may reduce apoptosis, protect mitochondrial function and slow myocardial aging. Recently, Yang et al (34) demonstrated that melatonin conferred a cardio-protective effect by improving post-ischemic cardiac function, decreasing infarct size, reducing the apoptotic index, diminishing release of lactate dehydrogenase, upregulating Bcl-2 and downregulating Bax.

In the present study, Bcl-2 expression was higher in the control group compared with the other groups, and in the melatonin-treated compared with untreated accelerated-aging groups. In addition, Bax protein levels were not significantly different among groups. However, the $\mathrm{Bcl}-2 / \mathrm{Bax}$ ratio in control and melatonin-treated groups was significantly higher than that in the untreated accelerated aging group. Release of cyt-c from the mitochondria to the cytoplasm is modulated by the Bcl-2 family, where Bcl-2 suppresses release of cyt-c, and Bax facilitates cyt-c release (44). The increase of $\mathrm{Bcl}-2 / \mathrm{Bax}$ in the melatonin-treated group implies reduced release of cyt-c in the mitochondria. This suggests that melatonin may reduce the release of cyt $\mathrm{c}$ from mitochondria to the cytoplasm via modulation of the Bcl-2 family. Melatonin modulates the expression of the Bcl-2 family, which may also prevent translocation of cyt-c from the mitochondria to the cytoplasm.

The results of the current study are limited by the small sample size and the short duration of study. In addition, chronic melatonin use was not evaluated. Rats were sacrificed early, which did not allow measurement of the effects of melatonin on rat survival and age-related changes in the heart over time. In addition, the molecular characteristics of melatonin such as its interaction with lipids and the stabilization of mitochondrial membranes were not examined. This may have enabled the elucidation of the mechanism of melatonin in protecting the mitochondria. In order to evaluate of the effects of long-term melatonin treatment, future studies will include a larger sample size with rats aged over 10 months that will be observed over a longer study period. The specific actions of melatonin on the electron transport chain and its complex activity will also be investigated, and its role in maintaining intramitochondrial homeostasis will be compared with other antioxidants (e.g. N-acetylcysteine).

In conclusion, the results of the present study suggest that melatonin exhibits a protective effect on mitochondrial function in a rat model of accelerated aging. Melatonin may maintain the electron transfer chain by increasing mitochondrial cyt-c levels, thus ensuring normal oxidative phosphorylation, maintaining ATP production and decreasing electron leakage and free radical production. The results of the current and future studies may increase our understanding of the anti-aging effects of melatonin on the mitochondria, thus providing more information about the aging process as well as strategies for anti-aging or treatments for age-associated diseases.

\section{Acknowledgements}

All authors appreciate the great contribution of Mrs. Shi-Wen Wang (Institute of Geriatric Cardiology, Chinese PLA General Hospital) to the manuscript before she passed away.

\section{References}

1. Harman D: The free radical theory of aging. Antioxid Redox Signal 5: 557-561, 2003.

2. Rodríguez MI, Carretero M, Escames G, López LC, Maldonado MD, Tan DX, Reiter RJ and Acuña-Castroviejo D: Chronic melatonin treatment prevents age-dependent cardiac mitochondrial dysfunction in senescence-accelerated mice. Free Radic Res 41: 15-24, 2007.

3. Takeda T: Senescence-accelerated mouse (SAM): A biogerontological resource in aging research. Neurobiol Aging 20: 105-110, 1999.

4. Rizvi SI and Jha R: Strategies for the discovery of anti-aging compounds. Expert Opin Drug Discov 6: 89-102, 2011.

5. Acuña-Castroviejo D, Martín M, Macías M, Escames G, León J, Khaldy H and Reiter RJ: Melatonin, mitochondria, and cellular bioenergetics. J Pineal Res 30: 65-74, 2001.

6. Rodriguez MI, Escames G, López LC, García JA, Ortiz F, López A and Acuña-Castroviejo D: Melatonin administration prevents cardiac and diaphragmatic mitochondrial oxidative damage in senescence-accelerated mice. J Endocrinol 194: 637-643, 2007.

7. Escames G, Lopez A, García JA, García L, Acuña-Castroviejo D, García JJ and López LC: The role of mitochondria in brain aging and the effects of melatonin. Curr Neuropharmacol 8: 182-193,2010.

8. Petrosillo G, Moro N, Paradies V, Ruggiero FM and Paradies G: Increased susceptibility to $\mathrm{Ca}(2+)$-induced permeability transition and to cytochrome $\mathrm{c}$ release in rat heart mitochondria with aging: Effect of melatonin. J Pineal Res 48: 340-346, 2010.

9. Judge $\mathrm{S}$ and Leeuwenburgh C: Cardiac mitochondrial bioenergetics, oxidative stress, and aging. Am J Physiol Cell Physiol 292: C1983-C1992, 2007.

10. Lesnefsky EJ, Moghaddas S, Tandler B, Kerner J and Hoppel CL: Mitochondrial dysfunction in cardiac disease: Ischemia-reperfusion, aging, and heart failure. J Mol Cell Cardiol 33: 1065-1089, 2001.

11. Lee SJ, Jin Y, Yoon HY, Choi BO, Kim HC, Oh YK, Kim HS and Kim WK: Ciclopirox protects mitochondria from hydrogen peroxide toxicity. Br J Pharmacol 145: 469-476, 2005.

12. Poeggeler B, Durand G, Polidori A, Pappolla MA, Vega-Naredo I, Coto-Montes A, Böker J, Hardeland R and Pucci B: Mitochondrial medicine: Neuroprotection and life extension by the new amphiphilic nitrone LPBNAH acting as a highly potent antioxidant agent. J Neurochem 95: 962-973, 2005.

13. Schapira AH: Mitochondrial involvement in Parkinson's disease, Huntington's disease, hereditary spastic paraplegia and Friedreich's ataxia. Biochim Biophys Acta 1410: 159-170, 1999.

14. Barlow-Walden LR, Reiter RJ, Abe M, Pablos M, Menendez-Pelaez A, Chen LD and Poeggeler B: Melatonin stimulates brain glutathione peroxidase activity. Neurochem Int 26: 497-502, 1995.

15. Tan DX, Chen LD, Poeggeler B, Manchester LC and Reiter RJ: Melatonin: A potent endogenous hydroxyl radical scavenger. Endocrine J 1: 57-60, 1993.

16. Hardeland R: Melatonin and the theories of aging: A critical appraisal of melatonin's role in antiaging mechanisms. J Pineal Res 55: 325-356, 2013.

17. Acuna-Castroviejo D, Escames G, Rodriguez MI and Lopez LC: Melatonin role in the mitochondrial function. Front Biosci 12: 947-963, 2007.

18. Reiter RJ: Oxidative processes and antioxidative defense mechanisms in the aging brain. FASEB J 9: 526-533, 1995.

19. Reiter RJ, Tan DX, Mayo JC, Sainz RM, Leon J and Czarnocki Z: Melatonin as an antioxidant: Biochemical mechanisms and pathophysiological implications in humans. Acta Biochim Pol 50: 1129-1146, 2003.

20. Okatani Y, Wakatsuki A, Shinohara K, Taniguchi K and Fukaya T: Melatonin protects against oxidative mitochondrial damage induced in rat placenta by ischemia and reperfusion. J Pineal Res 31: 173-178, 2001.

21. Okatani Y, Wakatsuki A, Reiter RJ and Miyahara Y: Melatonin reduces oxidative damage of neural lipids and proteins in senescence-accelerated mouse. Neurobiol Aging 23: 639-644, 2002.

22. Lardone PJ, Alvarez-García O, Carrillo-Vico A, Vega-Naredo I, Caballero B, Guerrero JM and Coto-Montes A: Inverse correlation between endogenous melatonin levels and oxidative damage in some tissues of SAM P8 mice. J Pineal Res 40: 153-157, 2006.

23. Bubenik GA and Konturek SJ: Melatonin and aging: Prospects for human treatment. J Physiol Pharmacol 62: 13-19, 2011. 
24. Reiter RJ, Richardson BA, Johnson LY, Ferguson BN and Dinh DT: Pineal melatonin rhythm: Reduction in aging Syrian hamsters. Science 210: 1372-1373, 1980.

25. Reiter RJ, Craft CM, Johnson JE Jr, King TS, Richardson BA, Vaughan GM and Vaughan MK: Age-associated reduction in nocturnal pineal melatonin levels in female rats. Endocrinology 109: 1295-1297, 1981.

26. Karasek M: Melatonin, human aging, and age-related diseases. Exp Gerontol 39: 1723-1729, 2004.

27. Wei H, Li L, Song Q, Ai H, Chu J and Li W: Behavioural study of the $\mathrm{D}$-galactose induced aging model in $\mathrm{C} 57 \mathrm{BL} / 6 \mathrm{~J}$ mice. Behav Brain Res 157: 245-251, 2005.

28. Song X, Bao M, Li D and Li YM: Advanced glycation in D-galactose induced mouse aging model. Mech Ageing Dev 108: 239-251, 1999.

29. Deng HB, Cheng CL, Cui DP, Li DD, Cui L and Cai NS: Structural and functional changes of immune system in aging mouse induced by D-galactose. Biomed Environ Sci 19: 432-438, 2006.

30. Hammond JB and Kruger NJ: The bradford method for protein quantitation. Methods Mol Biol 3: 25-32, 1988.

31. Khlyntseva SV, Bazel YR, Vishnikin AB and Andruch V: Methods for the determination of adenosine triphosphate and other adenine nucleotides. J Analytic Chem 64: 657-673, 2009.

32. Lee SD, Kuo WW, Ho YJ, Lin AC, Tsai CH, Wang HF, Kuo CH, Yang AL, Huang CY and Hwang JM: Cardiac Fas-dependent and mitochondria-dependent apoptosis in ovariectomized rats. Maturitas 61: 268-277, 2008.

33. Karyn M. Usher, Steven W. Hansen, Jennifer S. Amoo, Allison P. Bernstein and Mary Ellen P. McNally: Precision of internal standard and external standard methods in high performance liquid chromatography. LCGC Special Issues 33: 40-46, 2015.

34. Yang Y, Duan W, Jin Z, Yi W, Yan J, Zhang S, Wang N, Liang Z, Li Y, Chen W, et al: JAK2/STAT3 activation by melatonin attenuates the mitochondrial oxidative damage induced by myocardial ischemia/reperfusion injury. J Pineal Res 55: 275-286, 2013.
35. Carretero M, Escames G, López LC, Venegas C, Dayoub JC, García L and Acuña-Castroviejo D: Long-term melatonin administration protects brain mitochondria from aging. J Pineal Res 47: 192-200, 2009.

36. Martín M, Macías M, León J, Escames G, Khaldy H and Acuña-Castroviejo D: Melatonin increases the activity of the oxidative phosphorylation enzymes and the production of ATP in rat brain and liver mitochondria. Int J Biochem Cell Biol 34: 348-357, 2002.

37. Atkinson DE and Fall L: Adenosine triphosphate conservation in biosynthetic regulation. Escherichia coli phosphoribosylpyrophosphate synthase. J Biol Chem 242: 3241-3242, 1967.

38. Lopez LC, Escames G, Ortiz F, Ros E and Acuña-Castroviejo D: Melatonin restores the mitochondrial production of ATP in septic mice. Neuro Endocrinol Lett 27: 623-630, 2006.

39. Karasek M, Reiter RJ, Cardinali DP and Pawlikowski M: Future of melatonin as a therapeutic agent. Neuro Endocrinol Lett 23 (Suppl 1): S118-S121, 2002

40. Galano A, Tan DX and Reiter RJ: On the free radical scavenging activities of melatonin's metabolites, AFMK and AMK. J Pineal Res 54: 245-257, 2013.

41. Galano A, Tan DX and Reiter RJ: Melatonin as a natural ally against oxidative stress: A physicochemical examination. J Pineal Res 51: 1-16, 2011.

42. Green DR and Reed JC: Mitochondria and apoptosis. Science 281: 1309-1312, 1998.

43. Fang X, Chen M and Chen R: Cytochrome C and Apoptosis. J Foreign Medical Sciences (Clinical Biochemistry and Laboratory Medicine) 26: 43-46, 2005.

44. Shimizu S, Narita M and Tsujimoto Y: Bcl-2 family proteins regulate the release of apoptogenic cytochrome $\mathrm{c}$ by the mitochondrial channel VDAC. Nature 399: 483-487, 1999. 